\title{
Spontaneous polarization and piezoelectric constants of III-V nitrides
}

\author{
Fabio Bernardini and Vincenzo Fiorentini \\ INFM - Dipartimento di Scienze Fisiche, Università di Cagliari, I-09124 Cagliari, Italy \\ David Vanderbilt \\ Department of Physics and Astronomy, Rutgers University, Piscataway, NJ, U.S.A.
}

\begin{abstract}
The spontaneous polarization, dynamical Born charges, and piezoelectric constants of the III-V nitrides AlN, GaN, and InN are studied ab initio using the Berry phase approach to polarization in solids. The piezoelectric constants are found to be up 10 times larger than in conventional III-V's and II-VI's, and comparable to those of ZnO. Further properties at variance with those of conventional III-V compounds are the sign of the piezoelectric constants (positive as in II-VI's) and the very large spontaneous polarization.
\end{abstract}

PACS: 77.65.Bn, 77.84.Bw, 77.22.Ej

In this paper we report an ab initio study of the spontaneous polarization, piezoelectric constants, and dynamical charges of the III-V nitride semiconductors AlN, GaN, and InN. This class of polarization-related properties is of obvious importance for the study of nitride-based piezodevices 2 and multilayer structures. In particular, the knowledge of these properties allows an insightful treatment of the polarization (and ensuing electric fields) in strained and polarized nitride junctions and superlat ${ }_{\overline{-}}$ tices under any strain condition, as discussed elsewhere. 3 From the present study, one of the first peplications of the modern theory of polarization in solids, to real and "difficult" materials of technological interest (the first to our knowledge using pseudopotentials), the nitrides emerge as highly unusual III-V materials, resembling II-VI oxides and in some respects ferroelectric perovskites. The results we report here are of special interest in view of the scarcity of the data (both experimental and theoretical) available at present for the nitrides.

In the absence of external fields, the total macroscopic polarization $\mathbf{P}$ of a solid is the sum of the spontaneous polarization $\mathbf{P}^{\text {eq }}$ in the equilibrium structure, and of the strain-induced or piezoelectric polarization $\delta \mathbf{P}$. In the linear regime, the piezoelectric polarization is related to the strain $\epsilon$ by

$$
\delta P_{i}=\sum_{j} e_{i j} \epsilon_{j}
$$

which defines the components of the piezoelectric tensor $e_{i j}$ (Voigt notation is used).

The natural structure of the III-V nitrides is wurtzite, an hexagonal crystal structure defined by the edge length $a$ of the basal hexagon, the height $c$ of the hexagonal prism, and an internal parameter $u$ defined as the anioncation bond length along the (0001) axis in units of $c$. Wurtzite is the structure with highest symmetry compatible with the existence of spontaneous polarization, 60 and the piezoelectric tensor of wurtzite has three nonvanishing independent components. Therefore, the polarization in these materials system will both a spontaneous and a piezoelectric component.
In this work we shall restrict ourselves to polarizations along the (0001) axis; this is the direction along which both standard bulk materials and nitride superlattices are grown. The spontaneous polarization vector then points in the direction of the $c$ axis, $\mathbf{P}^{\text {eq }}=P^{\text {eq }} \hat{\mathbf{z}}$, and the piezoelectric polarization is simply expressed via the piezoelectric coefficients $e_{33}$ and $e_{13}$ as

$$
\delta P_{3}=e_{33} \epsilon_{3}+e_{31}\left(\epsilon_{1}+\epsilon_{2}\right),
$$

where $\epsilon_{3}=\left(c-c_{0}\right) / c_{0}$ is the strain along the $c$ axis, $\epsilon_{1}=\epsilon_{2}=\left(a-a_{0}\right) / a_{0}$ is the strain in the basal plane, $a_{0}$ and $c_{0}$ being the equilibrium values of the lattice parameters. The third independent component of the piezoelectric tensor, $e_{15}$, is related to the polarization induced by a shear strain, and will not be considered in this work.

To linear order, the change in polarization can also be expressed as

$$
\delta P_{3}=\frac{\partial P_{3}}{\partial a}\left(a-a_{0}\right)+\frac{\partial P_{3}}{\partial c}\left(c-c_{0}\right)+\frac{\partial P_{3}}{\partial u}\left(u-u_{0},\right.
$$

where $a_{0}, \mathrm{c}_{0}$, and $u_{0}$ are the equilibrium lattice constants and internal parameter. (Note that the parameters $a, c$ and $u$ are not independent, because of the condition of vanishing forces along the axis direction.) Once the polarization derivatives in Eq. 3 are known, the macroscopic piezoelectric tensor coefficients can be expressed as

$$
e_{33}=c_{0} \frac{\partial P_{3}}{\partial c}+\frac{4 e c_{0}}{\sqrt{3} a_{0}^{2}} Z^{*} \frac{d u}{d c}
$$

and

$$
e_{31}=\frac{a_{0}}{2} \frac{\partial P_{3}}{\partial a}+\frac{2 e}{\sqrt{3} a_{0}} Z^{*} \frac{d u}{d a}
$$

where

$$
Z^{*}=\frac{\sqrt{3} a_{0}^{2}}{4 e} \frac{\partial P_{3}}{\partial u} \equiv \mathcal{Z}_{3}^{(T)}
$$

is the axial component of the Born, or transverse, dynamical charge tensor $\mathcal{Z}^{(T)}$. In the last equation above, 
it is implicit that the vector connecting the cation with the anion has modulus $u c$ and points in the direction of the $c$ axis. The first term in Eqs. 14 and 5 is called the clamped-ion term, and it represents the effect of the strain on the electronic structure. The second term quantifies the effects of internal strain on the polarization. The derivatives of $u$ with respect to $c$ and $a$ in Eqs. 4 and 5 are related to the strain derivatives of $u$ through $c_{0} d u / d c=d u / d \epsilon_{3}$ and $a_{0} d u / d a=2 d u / d \epsilon_{1}$.

We have calculated the polarization within the Berry phase approach of Ref. A. The method's output is the difference in polarization among two states of a system, provided they can be connected by an adiabatic transformation which leaves the system insulating. The difference of electronic polarization $\boldsymbol{\Delta} \mathbf{P}_{\mathbf{e}}$ among two systems is then related to the geometric quantum phase by

$$
\Delta \mathbf{P}_{\mathbf{e}}=\mathbf{P}_{e}\left(\lambda_{2}\right)-\mathbf{P}_{e}\left(\lambda_{1}\right)
$$

where

$$
\mathbf{P}_{e}(\lambda)=-\left.\frac{2 e}{(2 \pi)^{3}} \int_{B Z} d \mathbf{k} \frac{\partial}{\partial \mathbf{k}^{\prime}} \phi^{(\lambda)}\left(\mathbf{k}, \mathbf{k}^{\prime}\right)\right|_{\mathbf{k}^{\prime}=\mathbf{k}},
$$

the integration domain in momentum space is the reciprocal unit cell, $\lambda$ characterizes the adiabatic transformation, and $\phi^{(\lambda)}$ is the geometric quantum phase

$$
\phi^{(\lambda)}\left(\mathbf{k}, \mathbf{k}^{\prime}\right)=\operatorname{Im}\left\{\ln \left[\operatorname{det}\left\langle u_{m}^{(\lambda)}(\mathbf{k}) \mid u_{n}^{(\lambda)}\left(\mathbf{k}^{\prime}\right)\right\rangle\right]\right.
$$

of the occupied crystal Bloch states $u_{n}^{(\lambda)}(\mathbf{k})$. The definition of the geometric quantum phase introduces an arbitrary constant in the value of the polarization so that the latter is well-defined only modulo $e \mathbf{R} / \Omega$, with $\mathbf{R}$ a realspace lattice vector. This is not a difficulty in practice, as one is generally interested in polarization changes such that $|\boldsymbol{\Delta} \mathbf{P}| \ll|e \mathbf{R} / \Omega|$, so that no ambiguity can arise.

The adiabatic transformation labeled by $\lambda$ is trivially identified as far as the strains $\epsilon_{1}, \epsilon_{2}$, and $\epsilon_{3}$ are concerned, so that the strain derivatives of the polarization within the wurtzite structure are easily calculated. On the other hand, the absolute value of the spontaneous polarization of the wurtzite structure (state $\lambda_{2}$ in Eq. 7) must be calculated as a difference relative to a structure having zero polarization, and functioning as reference state $\lambda_{1}$, since only polarization differences can be meaningfully calculated within the modern theory, $\mathrm{A}$ : zincblende is the natural choice for this purpose. One needs not actually devise a gap-preserving adiabatic transformation turning zincblende into wurtzite (a quite non-trivial task): the interface theorem of Ref. 8 proves that Eq. 0 can be used directly provided that an insulating interface can be built connecting the two different structures. As this is found to be the case 3 for the systems of relevance here, the absolute polarization of wurtzite nitrides can be easily computed (see also Ref. 7).

All the present first pringiples calculations are based on density functional theory in the local density approximation (LDA) for the exchange-correlation energy functional, for which we adopt the Ceperley-Alder 10 form as parametrized by Perdew and Zunger.11 The wave functions are expanded in a plane-wave basis set up toan energy cutoff of $25 \mathrm{Ry}$. Ultrasoft pseudopotentials 12 have been employed for all the elements involved in the calculations. In particular, the Ga and In pseudopotentials are constructed so as to explicitly include the semicore $d$ electrons in the valence, resulting in a very accurate description of the electronic and structural properties.

All calculations for the wurtzite structure used a mesh of 12 Chadi-Cohen special points for the Brillouin zone sampling. 13 The k-space integration for computing the polarization was instead done on a $(4,4,10)$ MonkhorstPack 14 mesh; this amounts to $16 \mathbf{k}_{\perp}$ points times $10 \mathbf{k}_{\|}$ points. Refining the mesh to 360 points (the $(6,6,10)$ mesh) gave no appreciable variation of the calculated values. The partial derivatives of the polarization appearing in Eq. 3 are easily evaluated numerically through the polarization change induced by typically a $\pm 2 \%$ change in the crystal parameters $a$ and $c$ (with $u$ kept fixed, i.e. clamped ion) and in the internal parameter $u$, around their equilibrium values.

Since we are interested in the spontaneous polarization, a careful determination of the equilibrium structure parameters is needed. The three independent structural parameters have been obtained by polynomial interpolation of the total energy values calculated over a grid of values of $a$ and $c$. The internal parameter $u$ was optimized at each $(a, c)$ grid point following the HellmannFeynman forces. The results, summarized in Table T, are very close to those reported in our previous work 19

In Table II we report the spontaneous polarization, dynamical charges, piezoelectric coefficients (and parts thereof) for the III-V nitrides. Also included are the same quantities calculated for $\mathrm{ZnO}$ and $\mathrm{BeO}$ (with the $\mathrm{Zn} 3 d$ states included in the valence). In addition, we list the same quantities as calculated in Ref. 16, using the Berry phase approach and the FLAPW method, for $\mathrm{ZnO}$ and $\mathrm{BeO}$. The agreement of our results with experiments for the latter materials is quite good. To facilitate further comparison with other III-V and II-VI systems, we have collected in Table III the piezoelectric constants for a number of III-V and II-VI compounds from Refs. 17, 18, and 19, calculated by density functional linear response theory. 20

To compare zincblende and wurtzite structures in Tables III and III, we have transformed the zincblende piezoelectric tensor by rotating to a coordinate system with $z$ along a (111) direction. Thus, $e_{33}=2 e_{14} / \sqrt{3}$ and $e_{31}=-e_{14} / \sqrt{3}$, with $e_{14}$ the piezoelectric constant of the zincblende material referred to the cubic axes. This direct comparison is meaningful since (1) the possible deviations from the ideal wurtzite structure do not influence significantly the polarization derivatives, and (2) it is known that in polytypical materials the values of the piezoelectric constants in the two competing structures agree to within a few percent (modulo the appropriate coordinate transformation) 16 
The III-V nitrides exhibit rather striking and unusual properties. Overall they resemble II-VI oxides, and $\mathrm{ZnO}$ in particular, and appear to be very different from conventional III-V semiconductors. Several features of the data in Tables III and III confirm this statement:

1) The absolute value of the piezoelectric constants is up to about 10 times larger than in conventional III-V's and II-VI's. In particular, both the constants $e_{33}$ and $e_{31}$ of AlN are larger than those of $\mathrm{ZnO}$, and are therefore the largest known so far among the tetrahedrally bonded semiconductors.

2) The spontaneous polarization, i.e. the polarization at zero strain, is very large in the nitrides. That of AlN, in particular, is the largest of our set of semiconductors, and only about 3-5-times smaller than in typical ferroelectric perovskites 21 Indeed, the piezoelectric constants themselves are only about 3 times smaller than in those ferroelectrics 22

3) The piezoelectric constants have the same sign as in IIVI compounds, and opposite to III-V's. While in normal III-V's the clamped-ion term is larger in absolute value than the internal-strain ionic contribution, in the nitrides the latter prevails due to the larger $Z^{*}$. Compared to normal III-V's, this sign inversion is a qualitative difference of obvious practical relevance. 23

4) The nitrides follow qualitatively a well defined III-V trend: the piezoelectric constants increase in magnitude as a function of the anion chemical identity as one moves upwards within Period V, i.e. from $\mathrm{Sb}$ to $\mathrm{N}$, because the ionic contribution tends to prevail over the electronic "clamped-ion" term as the anion becomes lighter (note that a similar trend is also obeyed by Zn II-VI's). The nitrides are an extreme case of this trend, and their piezoelectric response is by far larger than that of all other III-V's (see Table III), and of opposite sign.

5) The Born effective charge in the nitrides is within $10 \%$ of the nominal ionic charge, i.e. the ratio $Z^{*} / Z_{\text {ion }}$ is about unity. The same ratio is at most 0.7 in III-V compounds. In this context, it is appropriate to mention that the effective charges have no rigorous relation to nominal ionicity (although they may be a reasonable qualitative measure of ionicity in many binary compounds, suggesting trends analogous to those given by e.g. the charge asymmetry $g$ parameter of Garcia and Cohen 24) In particular, $Z^{*}$ needs not be smaller than or equal to the nominal ionic charge except in a purely ionic picture; conversely, whereas $Z^{*}<Z_{\text {ion }}$ may be interpreted as "smaller ionicity" in some sense, $Z^{*}>Z_{\text {ion }}$ is not meaningful in an ionic picture. Effective charges much larger than the nominal ionic charge are common in perovskites 21 and (to a lesser extent) even in alkaline earths oxides.25 This behavior is due to covalency and correlation contributions. We find (see Table II) $Z^{*}>Z_{\text {ion }}$ for InN, but with a deviation of $\sim 1 \%$, i.e. the effective charge is again very close to nominal ionicity.

Because of the sensitive dependence of the polarization on the structural parameters, there are some quantitative differences in spontaneous polarization for the three ni- trides. For instance, the increasing non-ideality of the structure going from GaN to InN to AlN ( $u$ gets longer and $c / a$ gets shorter), corresponds to an increase of the spontaneous polarization. To check this, we calculated the spontaneous polarization of AlN using the ideal structural parameters $u_{\mathrm{id}}=0.375$ and $c_{\mathrm{id}}=\sqrt{8 / 3} a$, and obtained a spontaneous polarization equal to $-0.033 \mathrm{C} / \mathrm{m}^{2}$, very similar to GaN and $\mathrm{InN}$, and a factor of 2.5 smaller than the spontaneous polarization in the actual equilibrium structure. The piezoelectric constants (i.e. polarization derivatives) are instead expected to vary mildly.

Additional III-V-like trends to be observed are that in III-V's the effective charge has similar values for AlAs and GaAs, but a rather larger value for InAs.17 This trend is clearly obeyed by the nitrides as well, indeed in a more clearcut way than in most other III-V's. Another interesting trend is the connection between the chemical nature of the cation and the piezoelectric constant. The piezpelectric constant for $\mathrm{Al}$ and In III-V's was found to be 17 consistently larger than the corresponding value for Ga compounds. This is also true of the nitrides, where the deviations are again larger. Since our calculations include the semicore $d$ states in the valence for both In and $\mathrm{Ga}$, this trend is not an artifact of the neglect of semicore states as hypothesized in Ref. 17.

While it is known that treating the semicore $d$ states as valence is necessary to obtain good structural properties for GaN and InN, 15,26 neither the spontaneous polarization nor the effective charges are influenced strongly. Using a Ga pseudopotential in which the $3 d$ states had been frozen in the core, we recalculated the spontaneous polarization and effective charges of GaN using the structural parameters given in Table il: the results are $P^{\mathrm{eq}}=-0.031$ $\mathrm{C} / \mathrm{m}^{2}$ and $Z^{*}=2.73$, showing that the direct influence of the $3 d$ states on the final polarization value is minor. This was to be expected since the $d$ bands in GaN are quite flat over most of the Brillouin zone, so that the overlap matrix (Eq. 9) is nearly the identity for those states, and its contribution to the Berry phase is small.

Experimental data to compare our predictions with are very scarce. No data is available for the spontaneous polarization, a quite elusive quantity in this respect; our calculated values will be a useful input for device modeling.2. 3 Also in the case of the piezoelectric constants, our predicted data should be of use to future experimental work. The only experimental data available 27 to our knowledge are for $\mathrm{AlN}, e_{33}^{\exp }=1.55$ $\mathrm{C} / \mathrm{m}^{2}$, and $e_{31}^{\exp }=-0.58 \mathrm{C} / \mathrm{m}^{2}$. Our predicted values of $1.46 \mathrm{C} / \mathrm{m}^{2}$ and $-0.60 \mathrm{C} / \mathrm{m}^{2}$ agree with experiment to within $\sim 5 \%$.

In summary, we have calculated the spontaneous polarization, dynamical charges, and piezoelectric constants of III-V nitrides by means of the Berry phase approach. The results show that III-V nitrides resemble II-VI compounds in terms of the sign of the spontaneous polarization and of the piezoelectric constants; the latter constants are much larger in absolute value than those of all III-V's and II-VI's, except for ZnO. The values of sponta- 
neous polarization are comparable to or larger than those of II-VI's; AlN has the largest value reported so far for any binary compound, and only a factor of 3 to 4 smaller than in typical perovskites. Some remnant of normal III-V-like behavior survives, embodied in the trends of the piezoelectric constants and effective charges when the chemical identity of the anion or cation changes.

V.F and F.B. acknowledge partial support by the European Community through Contract BRE2-CT93, and by CINECA Bologna through Supercomputing Grants. D.V. acknowledges support of ONR Grant N00014-97-10048 .

${ }^{1}$ F. A. Ponce and D. P. Bour, Nature 386, 351 (1997).

${ }^{2}$ A. Bykhovski, B. Gelmont, M. Shur, and A. Khan, J. Appl. Phys. 77, 1616 (1995).

${ }^{3}$ F. Bernardini, V. Fiorentini, and D. Vanderbilt, in ' $I I I-V$ Nitrides, edited by F. A. Ponce, T. D. Moustakas, I. Asaki, B. A. Monemar, MRS Proceedings, vol. 449, (Materials Research Society, Pittsburgh 1997); to be published.

${ }^{4}$ R.D. King-Smith and D. Vanderbilt, Phys. Rev. B 47, 1651 (1993).

${ }^{5}$ R. Resta, Rev. Mod. Phys. 66, 899 (1994).

${ }^{6}$ J. F. Nye, Physical properties of crystals (Oxford U.P., Oxford 1985).

${ }^{7}$ See M. Posternak, A. Baldereschi A. Catellani, and R. Resta, Phys. Rev. Lett. 64, 1777 (1990).

${ }^{8}$ D. Vanderbilt and R.D. King-Smith, Phys. Rev. B 48, 4442 (1993).

${ }^{9}$ R. Dreizler and E. K. U. Gross, Density-functional theory (Springer, New York 1990).

${ }^{10}$ D. M. Ceperley and B. J. Alder, Phys. Rev. Lett. 45, 556 (1980).

11 J. Perdew and A. Zunger, Phys. Rev. B 23, 5048 (1981).

12 D. Vanderbilt, Phys. Rev. 41, 7892 (1990).

13 D. J. Chadi and M. L. Cohen, Phys. Rev. B 8, 5747 (1973).

${ }^{14}$ H. J. Monkhorst and J. D. Pack, Phys. Rev. B 13, 5188 (1976).

15 A. Satta, V. Fiorentini, A. Bosin, F. Meloni and D. Vanderbilt, in Gallium Nitride and related compounds, edited by R. D. Dupuis, J. A. Edmond, F. Ponce, and S. Nakamura, MRS Proceedings, vol. 395, p. 515 (Materials Research Society, Pittsburgh 1996).

${ }^{16}$ A. Dal Corso, M. Posternak, R. Resta, and A. Baldereschi, Phys. Rev. B 50, 10715 (1994).

17 S. de Gironcoli, S. Baroni, and R. Resta, Phys. Rev. Lett. 62, 2853 (1989).

18 A. Dal Corso, R. Resta and S. Baroni, Phys. Rev. B 47, 16252 (1993).

19 S. de Gironcoli, S. Baroni, and R. Resta, Ferroelectrics 111, 19 (1990).

20 S. Baroni, P. Giannozzi, and A. Testa, Phys. Rev. Lett. 58, 1861 (1987).
${ }^{21}$ W. Zhong, R. D. King-Smith, and D. Vanderbilt, Phys. Rev. Lett. 72, 3618 (1994); R. Resta, M. Posternak, and A. Baldereschi, Phys. Rev. Lett. 70, 1010 (1993).

${ }^{22}$ K. M. Rabe and U. W. Waghmare, Phys. Rev. B 55, 6161 (1997).

${ }^{23} \mathrm{AlP}$ and InP have indeed 17 a positive, but very small, $e_{14}$, which is again related to larger-than-average $Z^{*}$.

24 A. Garcia and M. L. Cohen, Phys Rev. B 47, 4215 (1993); ibid., 4221.

${ }^{25}$ M. Posternak, R. Resta, H. Krakauer, and A. Baldereschi, to be published in Phys. Rev. B.

${ }^{26}$ See e.g V. Fiorentini, M. Methfessel, and M. Scheffler, Phys. Rev. B 47, 13353 (1993); V. Fiorentini, A. Satta, D. Vanderbilt, S. Massidda, and F.Meloni, in The Physics of Semiconductors, edited by D. J. Lockwood, (World Scientific, Singapore, 1995), p.137.

27 J. G. Gualtieri, J. A. Kosinski, and A. Ballato, IEEE Trans. Ultrason. Ferroelectrics Frequency Control UFFC-41, 53 (1994).

TABLE I. Structural parameters for AlN, GaN and InN.

\begin{tabular}{lccc}
\hline \hline & $a_{0}$ (bohr) & $c_{0} / a_{0}$ & $u_{0}$ \\
\hline AlN & 5.814 & 1.6190 & 0.380 \\
GaN & 6.040 & 1.6336 & 0.376 \\
$\mathrm{InN}$ & 6.660 & 1.6270 & 0.377 \\
\hline \hline
\end{tabular}

TABLE II. Calculated spontaneous polarization (in units of $\mathrm{C} / \mathrm{m}^{2}$ ), Born effective charges (in units of $e$ ), and piezoelectric constants (in units of $\mathrm{C} / \mathrm{m}^{2}$ ) for III- $\mathrm{V}$ wurtzite nitrides and II-VI wurtzite oxides.

\begin{tabular}{lccccccc}
\hline \hline & $P^{\mathrm{eq}}$ & $Z^{*}$ & $d u / d \epsilon_{3}$ & $e_{33}$ & $e_{31}$ & $e_{33}^{(0)}$ & $e_{31}^{(0)}$ \\
\hline $\mathrm{AlN}$ & -0.081 & -2.70 & -0.18 & 1.46 & -0.60 & -0.47 & 0.36 \\
$\mathrm{GaN}$ & -0.029 & -2.72 & -0.16 & 0.73 & -0.49 & -0.84 & 0.45 \\
$\mathrm{InN}$ & -0.032 & -3.02 & -0.20 & 0.97 & -0.57 & -0.88 & 0.45 \\
$\mathrm{ZnO}$ & -0.057 & -2.11 & -0.21 & 0.89 & -0.51 & -0.66 & 0.38 \\
$\mathrm{BeO}$ & -0.045 & -1.85 & -0.06 & -0.02 & -0.02 & -0.60 & 0.35 \\
\hline $\mathrm{ZnO}^{a}$ & -0.05 & -2.05 & -0.25 & 1.21 & -0.51 & -0.58 & 0.37 \\
$\mathrm{BeO}^{a}$ & -0.05 & -1.72 & -0.09 & 0.50 & - & -0.29 & - \\
\hline \hline
\end{tabular}

${ }^{a}$ Reference 16

TABLE III. Piezoelectric constants (in units of $\mathrm{C} / \mathrm{m}^{2}$ ) of several zincblende compounds, calculated via linear response theory in Refs. 17 (III-V), 18 (CdTe) and 19 (other II-VI).

\begin{tabular}{llllcl}
\hline \hline & $e_{33}$ & $e_{31}$ & & $e_{33}$ & $e_{31}$ \\
\hline $\mathrm{CdTe}$ & 0.03 & -0.01 & AlAs & -0.01 & 0.01 \\
$\mathrm{ZnS}$ & 0.10 & -0.05 & GaAs & -0.12 & 0.06 \\
$\mathrm{ZnSe}$ & 0.04 & -0.02 & InAs & -0.03 & 0.01 \\
$\mathrm{AlP}$ & 0.04 & -0.02 & $\mathrm{AlSb}$ & -0.04 & 0.02 \\
$\mathrm{GaP}$ & -0.07 & 0.03 & GaSb & -0.12 & 0.06 \\
$\mathrm{InP}$ & 0.04 & -0.02 & InSb & -0.06 & 0.03 \\
\hline \hline
\end{tabular}

